EUROPEAN JOURNAL OF PURE AND APPLIED MATHEMATICS

Vol. 14, No. 4, 2021, 1295-1305

ISSN 1307-5543 - ejpam.com

Published by New York Business Global

\title{
Definite integral of exponential polynomial and hyperbolic function in terms of the incomplete gamma function
}

\author{
Robert Reynolds ${ }^{1, *}$, Allan Stauffer ${ }^{1}$ \\ 1 Department of Mathematics and Statistics, Faculty of Science, York University, Toronto, \\ Ontario, Canada, M3J1P3
}

\begin{abstract}
This current paper is a table of definite integrals involving hyperbolic and logarithmic functions expressed in terms of the incomplete gamma function and fundamental constants. All the results in this work are new.
\end{abstract}

2020 Mathematics Subject Classifications: 30E20,33-01, 33-03, 33-04, 33-33B, 33E20

Key Words and Phrases: Entry in Oberhettinger, incomplete gamma function, definite integral, Euler's constant, contour integral

\section{Significance statement}

In 1990, Fritz Oberhettinger [5] published his book on Fourier transforms inter alia. In his book a vast number of definite integrals and transforms are summarized and tables produced. These tables are used in a wide area of mathematics namely probability and mathematical statistics [3], Fourier series for a combination of Jacobian elliptic functions [8], traveling wave solutions to the two-dimensional Korteweg-deVries equation [2], and fundamental solution of hyperbolic differential operators and the poisson summation formula [7] to name a few.

Since there is vast usage of integrals in the book of Oberhettinger [5], in this paper the authors aim to expand on these tables of integral formula involving exponential and hyperbolic functions by providing new formulae derived in this work. Another aim in this work is to provide the correct derivation of one of the integrals tables in [5], which is of importance since the book of Oberhettinger is so widely used. Providing an updated version of these tables will also assist in expanding current and future research where these types of integrals are used.

*Corresponding author.

DOI: https://doi.org/10.29020/nybg.ejpam.v14i4.4064

Email addresses: milver@my.yorku.ca (R. Reynolds), stauffer@yorku.ca (A. Stauffer) 


\section{Introduction}

In this paper we derive the definite integral given by

$$
\int_{0}^{\infty} \log (\tanh (\alpha x))\left(e^{i m x}(\log (a)+i x)^{k}+e^{-i m x}(\log (a)-i x)^{k}\right) d x
$$

where the parameters $k, a$ are general complex numbers, $\operatorname{Re}(\alpha)>0$ and $-1<\operatorname{Re}(m)<0$. The integral will be used to derive special cases in terms of special functions and fundamental constants. The derivations follow the method used by us in [9]. This method involves using a form of the generalized Cauchy's integral formula given by

$$
\frac{y^{k}}{\Gamma(k+1)}=\frac{1}{2 \pi i} \int_{C} \frac{e^{w y}}{w^{k+1}} d w .
$$

where $C$ is in general an open contour in the complex plane where the bilinear concomitant [9] has the same value at the end points of the contour. We then multiply both sides by a function of $x$ and then take a definite integral of both sides. This yields a definite integral in terms of a contour integral. Then we multiply both sides of equation (2) by another function of $x$ and take the infinite sums of both sides such that the contour integral of both equations are the same.

\section{Definite integral of the contour integral}

We use the method in [9]. The variable of integration in the contour integral is $t=$ $m+w$. The cut and contour are in the second quadrant of the complex $z$-plane. The cut approaches the origin from the interior of the first quadrant and the contour goes round the origin with zero radius and is on opposite sides of the cut. Using equation (2) we replace $y \rightarrow i y+\log (a)$ and multiply by $e^{i m y}$. Next we replace $y \rightarrow-y$ to form a second equation and add both to get

$$
\frac{\left(e^{i m y}(\log (a)+i y)^{k}+e^{-i m y}(\log (a)-i y)^{k}\right)}{\Gamma(k+1)}=\frac{1}{2 \pi i} \int_{C} 2 a^{w} w^{-k-1} \cos (y(m+w)) d w
$$

Next we replace $y \rightarrow x$, multiply both sides by $\frac{1}{2} \log (\tanh (\alpha x))$ and take the definite integral over $x \in[0, \infty)$ to get

$$
\begin{aligned}
\int_{0}^{\infty} & \frac{\log (\tanh (\alpha x))\left(e^{i m x}(\log (a)+i x)^{k}+e^{-i m x}(\log (a)-i x)^{k}\right)}{2 \Gamma(k+1)} d x \\
= & \frac{1}{2 \pi i} \int_{0}^{\infty} \int_{C} a^{w} w^{-k-1} \cos (x(m+w)) \log (\tanh (\alpha x)) d w d x \\
= & \frac{1}{2 \pi i} \int_{C} \int_{0}^{\infty} a^{w} w^{-k-1} \cos (x(m+w)) \log (\tanh (\alpha x)) d x d w \\
& -\frac{1}{2 \pi i} \int_{C} \frac{\pi a^{w} w^{-k-1} \tanh \left(\frac{\pi(m+w)}{4 \alpha}\right)}{2(m+w)} d w
\end{aligned}
$$


from equation (1.7.7.112) in [5] where $0<\operatorname{Re}(w+m)<1$. We are able to switch the order of integration over $w+m$ and $x$ using Fubini's theorem since the integrand is of bounded measure over the space $\mathbb{C} \times[0, \infty)$. Note the equation quoted in [5] is in error.

\section{The Incomplete Gamma Function and its contour integral representation}

The incomplete gamma functions [1], $\gamma(a, z)$ and $\Gamma(a, z)$, are defined by

$$
\gamma(a, z)=\int_{0}^{z} t^{a-1} e^{-t} d t
$$

and

$$
\Gamma(a, z)=\int_{z}^{\infty} t^{a-1} e^{-t} d t
$$

where $\operatorname{Re}(a)>0$. The incomplete gamma function has a recurrence relation given by

$$
\gamma(a, z)+\Gamma(a, z)=\Gamma(a)
$$

where $a \neq 0,-1,-2, \ldots$ The incomplete gamma function is continued analytically by

$$
\gamma\left(a, z e^{2 m \pi i}\right)=e^{2 \pi m i a} \gamma(a, z)
$$

and

$$
\Gamma\left(a, z e^{2 m \pi i}\right)=e^{2 \pi m i a} \Gamma(a, z)+\left(1-e^{2 \pi m i a}\right) \Gamma(a)
$$

where $m \in \mathbb{Z}, \gamma^{*}(a, z)=\frac{z^{-a}}{\Gamma(a)} \gamma(a, z)$ is entire in $z$ and $a$. When $z \neq 0, \Gamma(a, z)$ is an entire function of $a$ and $\gamma(a, z)$ is meromorphic with simple poles at $a=-n$ for $n=0,1,2, \ldots$ with residue $\frac{(-1)^{n}}{n !}$. These definitions are listed in Section 8.2(i) and (ii) in [1].

\subsection{Infinite sum of the contour integral}

Use equation (2) and multiply both sides by $e^{m y}$ and integrate over $y \in[-\infty, y)$ to get

$$
\frac{(-1)^{k} m^{-k} \Gamma(k+1)+y^{k}(-m y)^{-k}(\Gamma(k+1,-m y)-k \Gamma(k))}{m \Gamma(k+1)}=\frac{1}{2 \pi i} \int_{C} \frac{w^{-k-1} e^{y(m+w)}}{m+w} d w
$$

where $\operatorname{Im}(w+m)>0$. Next we multiply both sides by $e^{-m y}$ and replace $y \rightarrow \log (a)+$ $\frac{2 \pi(y+1)}{b}$ to get 


$$
\begin{aligned}
& \frac{1}{m \Gamma(k+1)} e^{-m\left(\log (a)+\frac{2 \pi(y+1)}{b}\right)}\left(\left(\log (a)+\frac{2 \pi(y+1)}{b}\right)^{k}\right. \\
& \left(-m\left(\log (a)+\frac{2 \pi(y+1)}{b}\right)\right)^{-k} \\
& \left.\left(\Gamma\left(k+1,-m\left(\frac{2 \pi(y+1)}{b}+\log (a)\right)\right)-k \Gamma(k)\right)+(-1)^{k} m^{-k} \Gamma(k+1)\right) \\
& =\frac{1}{2 \pi i} \int_{C} \frac{w^{-k-1} e^{w\left(\log (a)+\frac{2 \pi(y+1)}{b}\right)}}{m+w} d w
\end{aligned}
$$

Next we multiply both sides by $(-1)^{y} e^{\frac{2 \pi m(y+1)}{b}}$ and take the infinite sum over $y \in[0, \infty)$ and simplify in terms of the incomplete gamma function to get

$$
\begin{aligned}
& \sum_{y=0}^{\infty} \frac{\pi(-1)^{y} a^{-m}}{m \Gamma(k+1)}\left(\left(2 \log (a)+\frac{\pi(y+1)}{\alpha}\right)^{k}\right. \\
& \left(-\frac{m(2 \alpha \log (a)+\pi y+\pi)}{\alpha}\right)^{-k} \\
& \left.\left(\Gamma(k+1)-\Gamma\left(k+1,-m\left(\frac{\pi(y+1)}{2 \alpha}+\log (a)\right)\right)\right)+(-1)^{k+1} m^{-k} \Gamma(k+1)\right) \\
& =\frac{1}{2 \pi i} \sum_{y=0}^{\infty} \int_{C} \frac{(-1)^{y} a^{w} w^{-k-1} e^{\frac{2 \pi(y+1)(m+w)}{b}}}{m+w} d w \\
& =\frac{1}{2 \pi i} \int_{C} \sum_{y=0}^{\infty} \frac{(-1)^{y} a^{w} w^{-k-1} e^{\frac{2 \pi(y+1)(m+w)}{b}}}{m+w} d w \\
& =-\frac{1}{2 \pi i} \int_{C} \frac{\pi a^{w} w^{-k-1}\left(\tanh \left(\frac{\pi(m+w)}{4 \alpha}\right)+1\right)}{2(m+w)} d w
\end{aligned}
$$

from equation (1.232.1) in [4] where $\operatorname{Im}(w+m)>0$ in order for the sum to converge. 


\subsubsection{Derivation of the additional contour}

Use equation (2) and replace

$y \rightarrow \log (a)+y$ and multiply both sides by $e^{m y}$ and take the definite integral over $y \in[0, \infty)$ to get

$$
\frac{\pi a^{-m}(-m)^{-k-1} \Gamma(k+1,-m \log (a))}{2 \Gamma(k+1)}=-\frac{1}{2 \pi i} \int_{C} \frac{\pi a^{w} w^{-k-1}}{2(m+w)} d w
$$

where $-1<\operatorname{Re}(m)<0$.

\section{Definite integral in terms of the incomplete gamma function}

Theorem 1. For $-1<\operatorname{Re}(m)<0, k, a \in \mathbb{C}, \operatorname{Re}(\alpha)>0$

$$
\begin{aligned}
& \int_{0}^{\infty} \log (\tanh (\alpha x))\left(e^{i m x}(\log (a)+i x)^{k}+e^{-i m x}(\log (a)-i x)^{k}\right) d x \\
& =\sum_{y=0}^{\infty} \frac{2 \pi(-1)^{y} a^{-m}}{m}\left(\left(2 \log (a)+\frac{\pi(y+1)}{\alpha}\right)^{k}\right. \\
& \left(-\frac{m(2 \alpha \log (a)+\pi y+\pi)}{\alpha}\right)^{-k} \\
& \left.\left(\Gamma(k+1)-\Gamma\left(k+1,-m\left(\frac{\pi(y+1)}{2 \alpha}+\log (a)\right)\right)\right)+(-1)^{k+1} m^{-k} \Gamma(k+1)\right) \\
& +\pi\left(-a^{-m}\right)(-m)^{-k-1} \Gamma(k+1,-m \log (a))
\end{aligned}
$$

Proof. Since the right-hand side of equation (4) is equal to the sum of the right-hand sides of equations (12) and (13) we can equate the left-hand sides to get the stated result.

\section{Table of definite integrals}

In this section we will evaluate equation (14) for various values of the parameters and express these integrals in terms of fundamental constants where possible. Some special functions and fundamental constants which occur in this section are Euler's constant $\gamma$ section (8.367) in [4], exponential integral function $E_{i}(x)$ equation (5.231.1) in [4], the imaginary error function $\operatorname{erf} i(i z) / i$ see equation (8.253.1) in [4] and equation (40:12:2) in [6]. 


\subsection{Derivation of entry 1.7.7.112 in [5]}

Lemma 1.

$$
\int_{0}^{\infty} \cos (m x) \log (\tanh (\alpha x)) d x=-\frac{\pi \tanh \left(\frac{\pi m}{4 \alpha}\right)}{2 m}
$$

Proof. Use equation (14) and set $k=0$ and simplify. Note the equation quoted in [5] is in error.

Proposition 1.

$$
\begin{aligned}
& \int_{0}^{\infty} x^{k}\left((-1)^{-k} e^{-i m x}+e^{i m x}\right) \log (\tanh (\alpha x)) d x \\
& =\sum_{y=0}^{\infty} \frac{2 \pi i^{-k}(-1)^{y}}{m}\left((-1)^{k+1} m^{-k} \Gamma(k+1)\right. \\
& \left.\quad+(-m)^{-k}\left(\Gamma(k+1)-\Gamma\left(k+1,-\frac{m \pi(y+1)}{2 \alpha}\right)\right)\right) \\
& +\pi\left(-i^{-k}\right)(-m)^{-k-1} \Gamma(k+1)
\end{aligned}
$$

Proof. Use equation (14) and set $a=1$ and simplify.

\section{Proposition 2.}

$$
\begin{aligned}
& \int_{0}^{\infty} \frac{\log (\tanh (\alpha x))(\log (a) \cos (m x)+x \sin (m x))}{\log ^{2}(a)+x^{2}} d x \\
& \quad=\pi \sum_{y=0}^{\infty}(-1)^{y} a^{-m}\left(\log \left(-\frac{m(2 \alpha \log (a)+\pi y+\pi)}{\alpha}\right)\right. \\
& \quad+\Gamma\left(0,-\frac{m(\pi y+2 \alpha \log (a)+\pi)}{2 \alpha}\right) \\
& \left.\quad-\log \left(2 \log (a)+\frac{\pi(y+1)}{\alpha}\right)-\log (m)+i \pi\right)-\frac{1}{2} \pi a^{-m} \Gamma(0,-m \log (a))
\end{aligned}
$$

Proof. Use equation (14) and apply L'Hopital's rule as $k \rightarrow-1$ and simplify.

\section{Lemma 2.}

$$
\begin{aligned}
& \int_{0}^{\infty} \frac{\log (\tanh (3 x))\left(\log (2) \cos \left(\frac{3 x}{4}\right)-x \sin \left(\frac{3 x}{4}\right)\right)}{x^{2}+\log ^{2}(2)} d x \\
& \quad=2^{3 / 4} \pi \sum_{y=0}^{\infty}(-1)^{y} \Gamma\left(0, \frac{1}{8}(\pi y+\log (64)+\pi)\right)+\frac{\pi E i\left(-\frac{3 \log (2)}{4}\right)}{\sqrt[4]{2}}
\end{aligned}
$$


Proof. Use equation (17) and set $a=2, \alpha=3, m=-3 / 4$ and simplify.

\section{Lemma 3.}

$$
\begin{aligned}
& \int_{0}^{\infty} \frac{\log (\tanh (3 x))\left(x \sin \left(\frac{3 x}{4}\right)-i \pi \cos \left(\frac{3 x}{4}\right)\right)}{\pi^{2}-x^{2}} d x \\
& \quad=\pi \sum_{y=0}^{\infty}(-1)^{y+\frac{3}{4}} \Gamma\left(0, \frac{1}{8} \pi(y+(1+6 i))\right) \\
& \quad-\frac{1}{2}(-1)^{3 / 4} \pi \Gamma\left(0, \frac{3 i \pi}{4}\right)
\end{aligned}
$$

Proof. Use equation (17) and set $a=-1, \alpha=3, m=-3 / 4$ and simplify.

\section{Lemma 4.}

$$
\begin{aligned}
& \int_{0}^{\infty} \frac{\log (\tanh (x))\left(\cos \left(\frac{2 x}{3}\right)-x \sin \left(\frac{2 x}{3}\right)\right)}{x^{2}+1} d x \\
& =e^{2 / 3} \pi \sum_{y=0}^{\infty}(-1)^{y} \Gamma\left(0, \frac{1}{3}(\pi y+\pi+2)\right) \\
& \quad+\frac{1}{2} e^{2 / 3} \pi E i\left(-\frac{2}{3}\right)
\end{aligned}
$$

Proof. Use equation (17) and set $a=e, \alpha=1, m=-2 / 3$ and simplify.

\section{Proposition 3.}

$$
\begin{aligned}
& \int_{0}^{\infty}(x \sin (m x)-i \pi \cos (m x)) \log (\tanh (\alpha x)) d x \\
& \quad=\sum_{y=0}^{\infty} \frac{\pi(-1)^{-m+y+1} \Gamma\left(2,-\frac{m \pi(y+2 i \alpha+1)}{2 \alpha}\right)}{m^{2}}+\frac{\pi(1-i \pi m)}{2 m^{2}}
\end{aligned}
$$

Proof. Use equation (14) and set $k=1, a=-1$ and simplify.

\section{Proposition 4.}

$$
\begin{aligned}
& \int_{0}^{\infty} e^{-\frac{2 i x}{3}}\left(e^{\frac{4 i x}{3}} \log (i(\pi-x))+\log (i(x+\pi))\right) \log (\tanh (\alpha x)) d x \\
& =\frac{3}{2} \pi \sum_{y=0}^{\infty}(-1)^{y}(i(\sqrt{3}+i) \\
& E_{1}\left(\frac{\pi(y+2 i \alpha+1)}{3 \alpha}\right) \\
& \left.+2 e^{-\frac{\pi(y+1)}{3 \alpha}} \log \left(\frac{\pi(2 i \alpha+y+1)}{2 \alpha}\right)\right) \\
& -\frac{3}{2} \pi\left(\log (i \pi)+(-1)^{2 / 3} \Gamma\left(0, \frac{2 i \pi}{3}\right)\right)
\end{aligned}
$$


Proof. Use equation (14) take the first partial derivative with respect to $k$ then set $k=0, m=-2 / 3, a=-1$ and simplify.

\section{Lemma 5.}

$$
\begin{aligned}
\int_{0}^{\infty} \frac{\log (\tanh (3 x))\left(\cos \left(\frac{2 x}{3}\right)-\sin \left(\frac{2 x}{3}\right)\right)}{\sqrt{x}} d x & \\
& =\sqrt{3} \pi \sum_{y=0}^{\infty}(-1)^{y} \Gamma\left(\frac{1}{2}, \frac{1}{9} \pi(y+1)\right)-\frac{1}{2} \sqrt{3} \pi^{3 / 2}
\end{aligned}
$$

Proof. Use equation (14) and set $k=-1 / 2, a=1, m=-2 / 3, \alpha=3$ and simplify.

\section{Lemma 6.}

$$
\begin{aligned}
& \int_{0}^{\infty} \frac{(\sin (m x)+\cos (m x)) \log (\tanh (\alpha x))}{\sqrt{x}} d x \\
& =\sum_{y=0}^{\infty} \frac{\sqrt{2} \pi^{3 / 2}(-1)^{y+1}\left(e r f i\left(\sqrt{\frac{\pi}{2}} \sqrt{m} \sqrt{\frac{y+1}{\alpha}}\right)-i\right)}{\sqrt{m}}+\frac{\pi^{3 / 2} m}{\sqrt{2}(-m)^{3 / 2}}
\end{aligned}
$$

Proof. Use equation (14) and set $k=-1 / 2, a=1$ and simplify.

\section{Lemma 7.}

$$
\begin{aligned}
\int_{0}^{\infty} \frac{\log (\tanh (x))\left(\cos \left(\frac{x}{2}\right)-\sin \left(\frac{x}{2}\right)\right)}{\sqrt{x}} d x & \\
& =2 \pi \sum_{y=0}^{\infty}(-1)^{y} \Gamma\left(\frac{1}{2}, \frac{1}{4} \pi(y+1)\right)-\pi^{3 / 2}
\end{aligned}
$$

Proof. Use equation (14) and set $k=-1 / 2, a=1, m=-1 / 2, \alpha=1$ and simplify.

\section{Lemma 8.}

$$
\begin{aligned}
& \int_{0}^{\infty} \sqrt{x} \log (\tanh (x))\left(\sin \left(\frac{x}{2}\right)\right. \\
& \left.+\cos \left(\frac{x}{2}\right)\right) d x \\
& =4 \pi \sum_{y=0}^{\infty}(-1)^{y} \Gamma\left(\frac{3}{2}, \frac{1}{4} \pi(y+1)\right)-\pi^{3 / 2}
\end{aligned}
$$

Proof. Use equation (14) and set $k=1 / 2, a=1, m=-1 / 2, \alpha=1$ and simplify. 
Lemma 9.

$$
\begin{aligned}
& \int_{0}^{\infty} \frac{e^{-\frac{i x}{2}}\left(\sqrt{\pi-x}+e^{i x} \sqrt{x+\pi}\right) \log (\tanh (\pi x))}{\sqrt{\pi^{2}-x^{2}}} d x \\
& =2 i \sqrt{2} \pi \sum_{y=0}^{\infty}(-1)^{y+\frac{1}{4}} \Gamma\left(\frac{1}{2}, \frac{1}{4}(y+2 i \pi+1)\right) \\
& \quad+(1-i) \pi \Gamma\left(\frac{1}{2}, \frac{i \pi}{2}\right)
\end{aligned}
$$

Proof. Use equation (14) and set $k=-1 / 2, a=-1, m=-1 / 2, \alpha=\pi$ and simplify.

Lemma 10.

$$
\begin{aligned}
& \int_{0}^{\infty} e^{-\frac{i x}{2}}\left(e^{i x} \log (-i x)+\log (i x)\right) \log (\tanh (\pi x)) d x \\
& =4 \pi \sum_{y=0}^{\infty}(-1)^{y}\left(e^{-\frac{y}{4}-\frac{1}{4}} \log \left(\frac{y+1}{2}\right)\right. \\
& \left.+\Gamma\left(0, \frac{y+1}{4}\right)\right)+2 \pi(\gamma-\log (2))
\end{aligned}
$$

Proof. Use equation (14) and set $a=1, m=-1 / 2, \alpha=\pi$ then take the first partial derivative with respect to $k$ and set $k=0$ and simplify.

\section{Lemma 11.}

$$
\begin{aligned}
& \int_{0}^{\infty} e^{\frac{i x}{2}}\left(\frac{e^{-i x}}{\sqrt[3]{i(x+\pi)}}+\frac{1}{\sqrt[3]{i(\pi-x)}}\right) \log (\tanh (2 \pi x)) d x \\
& =2 i 2^{2 / 3} \pi \sum_{y=0}^{\infty}(-1)^{y} \Gamma\left(\frac{2}{3}, \frac{1}{8}(y+4 i \pi+1)\right) \\
& -i 2^{2 / 3} \pi \Gamma\left(\frac{2}{3}, \frac{i \pi}{2}\right)
\end{aligned}
$$

Proof. Use equation (14) and set $k=-1 / 3, a=-1, \alpha=2 \pi, m=-1 / 2$ and simplify.

Lemma 12.

$$
\begin{aligned}
& \int_{0}^{\infty} e^{-\frac{i x}{2}}\left(-\frac{1}{(x+\pi)^{2}}-\frac{e^{i x}}{(\pi-x)^{2}}\right) \log (\tanh (2 \pi x)) d x \\
& =i \pi \sum_{y=0}^{\infty}(-1)^{y} \Gamma\left(-1, \frac{1}{8}(y+4 i \pi+1)\right) \\
& -E_{2}\left(\frac{i \pi}{2}\right)
\end{aligned}
$$


Proof. Use equation (14) set $a=-1, \alpha=2 \pi, m=-1 / 2$ and apply L'Hopital's rule as $k \rightarrow-2$ and simplify.

\section{Lemma 13.}

$$
\begin{aligned}
& \int_{0}^{\infty} i e^{-\frac{i x}{2}}\left(\frac{1}{(x+\pi)^{3}}+\frac{e^{i x}}{(\pi-x)^{3}}\right) \log (\tanh (3 \pi x)) d x \\
& =\frac{1}{2} i \pi \sum_{y=0}^{\infty}(-1)^{y} \Gamma\left(-2, \frac{1}{12}(y+6 i \pi+1)\right)+\frac{i E_{3}\left(\frac{i \pi}{2}\right)}{\pi}
\end{aligned}
$$

Proof. Use equation (14) set $a=-1, \alpha=2 \pi, m=-1 / 2$ and apply L'Hopital's rule as $k \rightarrow-3$ and simplify.

\section{Discussion}

In this work the authors derived new definite integral formulae involving exponential and hyperbolic functions and expressed this integral in terms of the incomplete gamma function. The authors also derived the correct version for an integral in the [5]. Using this derived integral formula we derived special cases in terms of fundamental constants and special functions. The authors used their contour integral method to derive the integral formula expressed in terms of the incomplete gamma function. The results presented were numerically verified for both real and imaginary and complex values of the parameters in the integrals using Mathematica by Wolfram.

\section{References}

[1] Nist digital library of mathematical functions. "F. W. J. Olver, A. B. Olde Daalhuis, D. W. Lozier, B. I. Schneider, R. F. Boisvert, C. W. Clark, B. R. Miller, B. V. Saunders, H. S. Cohl, and M. A. McClain, eds".

[2] Yunkai Chen and Shih-Liang Wen. Traveling wave solutions to the two-dimensional korteweg-devries equation. Journal of Mathematical Analysis and Applications, 127:226-236, 101987.

[3] Narayan C. Giri. Multivariate Statistical Inference. Academic Press, 1977.

[4] I. S. Gradshteyn and I. M. Ryzhik. Table of Integrals, Series, and Products. Academic Press, 052014.

[5] Fritz Oberhettinger. Tables of Fourier Transforms and Fourier Transforms of Distributions. Springer Berlin Heidelberg, 041990.

[6] Keith B. Oldham, Jan Myland, and Jerome Spanier. An Atlas of Functions: with Equator, the Atlas Function Calculator. Springer Science \& Business Media, 072010. 
[7] Norbert Ortner and Peter Wagner. Fundamental solution of hyperbolic differential operators and the poisson summation formula. Integral Transforms and Special Functions, 1:183-196, 121993.

[8] N. R. Pereira. Fourier series for a combination of jacobian elliptic functions: Problem 79-9. SIAM Review, 22:232-234, 1980.

[9] Robert Reynolds and Allan Stauffer. A method for evaluating definite integrals in terms of special functions with examples. International Mathematical Forum, 15:235$244,2020$. 